\title{
Teste de Valsalva ortostático no refluxo venoso
}

\author{
U pright Valsalva's test in venous reflux
}

\section{Gilberto G onçalves de Souza' ${ }^{1}$ Adamastor H umberto Pereira2, Luiz Francisco M achado da C osta², Edson Gassen ${ }^{3}$, Cláudio N huch ${ }^{3}$, Régis Renosto ${ }^{4}$, André Silvio Schier ${ }^{4}$, Leonardo Reis de Souza ${ }^{4}$}

\begin{abstract}
Resumo
O bjetivo: D eterminar se a modificação proposta pel os autores no teste de Valsal va proporcionou ganho de sensibilidade, especificidade e acurácia no diagnóstico do refluxo em relação ao teste realizado na posição supina, quando comparados com a flebografia, utilizada como padrão-ouro.

M étodo: $C$ ento e sessenta e um pacientes com insuficiência venosa crônica $\left(\mathrm{C}_{4,5}\right.$ ou $\left.{ }_{6} \mathrm{E}_{\mathrm{p}} \mathrm{A}_{S \mathrm{P} P} \mathrm{P}_{\mathrm{R}}\right)$ foram examinados prospectivamente. 0 s pacientes foram submetidos à avaliação hemodinâmica, incluindo o teste de Val salva supino padrão e o novo teste de Val sal va ortostático. A flebografia descendente foi utilizada como padrão-ouro, com a qual os testes foram comparados. Foi criada uma curva ROC (Receiver 0 perator Characteristic) para determinação do limite superior da normalidade do novo teste. 0 s valores obtidos para sensibilidade, especificidade e acurácia de cada um dos testes foram comparados entre si, utilizando o teste de comparação de porcentagens de D unn. Foi adotado o nível de significância de $5 \%$.

Resultados: 0 teste de Val sal va supino padrão atingiu uma sensibilidade de $65 \%$, especificidade de $81 \%$ e acurácia de $66 \%$, enquanto o teste de Valsalva ortostático atingiu uma sensibilidade de $80 \%$ $(P<0,01)$, especificidade de $63 \%(P=0,43)$ e acurácia de $78 \%$ $(P<0,05)$.

Conclusão: A modificação no teste de Valsalva proposta pelos autores determinou melhor sensibilidade e acurácia, sem decréscimo significante na especificidade, provando ser útil na avaliação do refluxo da insuficiência venosa crônica.
\end{abstract}

Palavras-chave: insuficiência venosa, manobra de Valsalva, diagnóstico.

\begin{abstract}
O bjective: To test sensitivity, specificity and accuracy of the modified upright Valsalva's test described by the authors for diagnosing the reflux, when compared to phlebography, which is used as gold standard.
\end{abstract}

M ethod: $O$ ne hundred sixty one patients with chronic venous insufficiency $\left(C_{4,5}\right.$ or $\left.{ }_{6} E_{P} A_{S D P} P_{R}\right)$ were prospectively examined. $T$ he patients were submitted to hemodynamic evaluation, including the standard supine Valsalva's test and the new modified upright Valsalva's test. D escending phlebography was used as a gold standard, to which the hemodynamic tests were compared. A RO C curve (Receiver $O$ perator $C$ haracteristic) was plotted to determine the upper limit of normality for the new test. The observed sensitivity, specificity and accuracy values of each test were compared using Dunn's test. The significance level of $5 \%$ was adopted.

Results: T he standard supine Val salva's test reached a sensitivity of $65 \%$, specificity of $81 \%$ and accuracy of $66 \%$, whereas the upright Valsalva's test reached a sensitivity of $80 \%(P<0.01)$, specificity of $63 \%(P=0.43)$ and accuracy of $78 \%(P<0.05)$.

Conclusion: The modification in the Valsalva's test proposed by the authors seems to determine better sensitivity and accuracy without significant decrease in specificity, proving to be useful in reflux evaluation of chronic venous insufficiency.

Key words: venous insufficiency, Valsalva maneuver, diagnosis.

1. Cirurgião vascular, Serviço de Cirurgia Vascular, Hospital de Clínicas de Porto Alegre (HCPA), Universidade Federal do Rio Grande do Sul (UFRGS), Porto Alegre, RS.

2. Professor adjunto, UFRGS, Porto Alegre, RS.

3. Médico residente, Serviço de Cirurgia Vascular, HCPA, Porto Alegre, RS

4. Acadêmico de Medicina, UFRGS, Porto Alegre, RS.

Artigo submetido em 30.06.05, aceito em 17.08.05. 
A hemodinâmica venosa éestudada desdea primeira metade do século 20. Em 1949, W arren, W hite e B elcher descreveram pressões no sistema da veia safena em extremidades normais, varicosas e pós-flebíticas ${ }^{1}$. $\mathrm{N}$ o mesmo ano, Pollak e W ood descreveram as variações que a pressão venosa sofre de acordo com mudanças na postura e com exercícios². Arnoldi, em 1966, descreveu uma rotina de avaliação da pressão venosa que ainda é usada ${ }^{3}$. Raju \& Fredericks, em 1990, descreveram um teste venoso hemodinâmico, inicialmente chamado de Elevação da Pressão V enosa do Pé, $V$ alsalva induzida ou teste de V alsalva supino (TVS) ${ }^{4}$. Essesautores sugeriram queessetesterefleteaspectos do refluxo não aferidos pela medida ambulatorial tradicional da pressão venosa e, desde então, tornou-se parte da avaliação venosa usada no $\mathrm{H}$ ospital de Clínicas de Porto Alegre (HCPA). 0 teste foi descrito para ser realizado com o paciente na posição supina.

A flebografia descendente deve ser realizada com o paciente na posição ortostática, na qual há melhor detecção do refluxo ${ }^{5-7}$. Do mesmo modo, o exame dúplex foi mais acurado quando executado na posição ortostática. Van Bemmelen et al. observaram que as velocidades do refluxo são menores quando o paciente está na posição supina, resultando em fechamento valvar anormal e tempo de refluxo falsamente elevado ${ }^{8,9}$. M asuda et al. sugeriram quea posição ortostática é melhor na detecção do refluxo pela técnica do manguito, mas não na técnica de Valsalva ${ }^{10}$.

0 objetivo do presente estudo é determinar se a modificação proposta pel os autores no teste deV alsal va determina ganho de sensibilidade, especificidadee acurácia no diagnóstico do refluxo em relação ao teste realizado na posição supina, quando comparados com a flebografia, utilizada como padrão-ouro.

\section{M étodo}

Cento e sessenta e um pacientes com insuficiência venosa crônica grave, com doença primária (os pacientes com doença refluxiva pós-trombótica foram excluídos) e refluxo no sistema superficial, profundo ou perfurante $\left(C_{4,5} \text { ou }{ }_{6} E_{P} A_{S D} P_{R}\right)^{11}$ foram examinados, consecutivamente, no setor de cirurgia vascular do HCPA, sendo submetidos à avaliação hemodinâmica, flebografia ascendente e descendente pelas técnicas descritas previamente em outros artigos $5,12,13$. Os pacientes com doença obstrutiva também foram excluídos do estudo. A avaliação hemodinâmica venosa foi realizada inserindo agulha em veia no dorso do pé, conectada a transdutor de pressão, com os dados gravados e analisados por sistema computadorizado desenvolvido pelo Serviço de Engenharia Biomédica do HCPA. A avaliação hemodinâmica incluiu o TVS e o teste de Valsalva ortostático proposto (TV O ). A única modificação da técnica foi a posição do paciente, e o exame foi real izado com o paciente soprando contra manômetro, atingindo pressão de $40 \mathrm{mmH}$ g. A flebografia descendente foi usada como o padrão-ouro para 0 refluxo. Foi considerada negativa (grau 0), quando não havia refluxo; grau 1, quando o refluxo estava restrito ao terço proximal da coxa; grau 2, quando 0 refluxo chegava até o terço distal da coxa; grau 3, quando 0 refluxo atingia a veia poplítea distal ao joelho; e grau 4, quando o refluxo atingia tributárias da veia poplítea. A participação de sistemas múltiplos (profundo, superficial) e níveis múltiplos (proximal e distal) de refluxo foram considerados como proposto por R aju ${ }^{14}$ e correlacionados com o TVS e o T V 0 . 0 refluxo em múltiplos sistemas e múltiplos níveis parece ser necessário, na maioria dos pacientes, para produzir sintomas ${ }^{15}$.

Para determinar o limite superior a ser considerado como normal para o novo TVO, uma curva ROC (Receiver 0 perator Characteristic) foi observada. $O$ utra curva RO C, para comparação, foi criada para o TVS padrão. Sensibilidade, especificidade e acurácia foram calculadas para os dois testes nos pontos de corte propostos, eosval oresobtidoscomparadospel o testede comparação de porcentagens de D unn. Foi observado o intervalo de confiança de $95 \%$ dos dois testes nos pontos de corte propostos. Foi adotado o nível de significância de $5 \% 16$.

Todos os pacientes foram informados sobre as técnicas diagnósticas. Astécnicasadotadasnainvestigação de insuficiência venosa crônica foram submetidase aprovadas pelo G rupo de Pesquisa ePós-G raduação da Universidade Federal do Rio Grande do Sul e pelo Comitê de Pesquisa da mesma instituição.

\section{Resultados}

A análise da curva ROC do TVO (Figura 1) mostrou que o mel hor ponto de corte é $20 \mathrm{mmH}$ g, com resultados $\geq 21 \mathrm{mmH}$ g sendo compatíveis com refluxo. $\mathrm{N}$ esse ponto de corte de $20 \mathrm{mmH}$ g, a sensibilidade é de $80 \%$, a especificidade de $63 \%$ e a acurácia de $78 \%$. 0 bservand o a curva do T VS (Figu- 


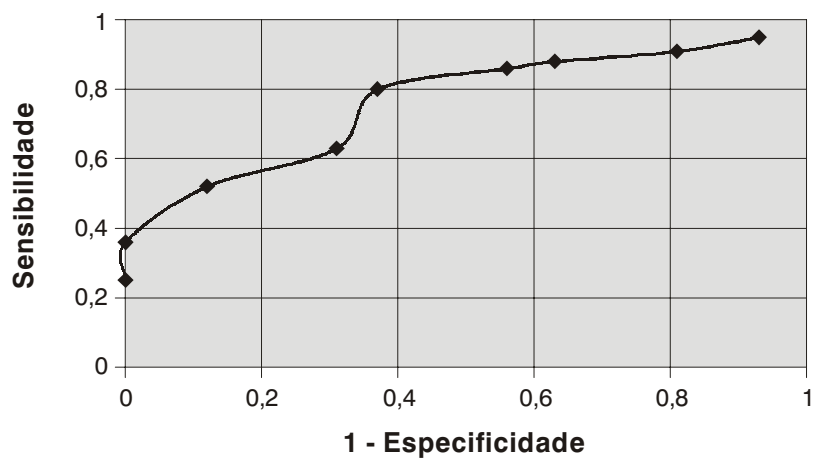

* $20 \mathrm{mmHg}$ para sensibilidade de $80 \%$ e especificidade de $63 \%$.

Figura 1 - Teste ROC para V al salva ortostática

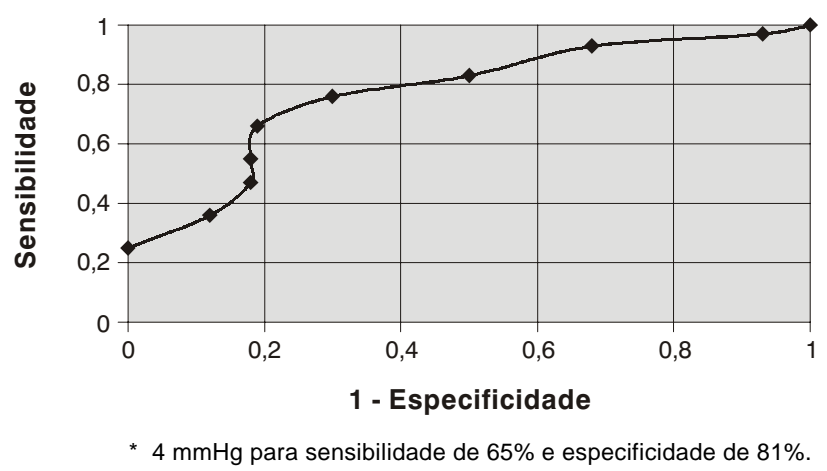

Figura 2 - T este ROC para V al sal va padrão

ra 2), o ponto de corte proposto - $4 \mathrm{mmH} \mathrm{g} \mathrm{-} \mathrm{é}$ confirmado, alcançando uma sensibilidade de $65 \%$, especificidade de $81 \%$ e acurácia de $66 \%$. U sando 0 teste de comparação de porcentagens de Dunn, a sensibilidade do TVO é significativamente melhor $(P=0,003847)$, a especificidade não é estatisticamente diferente ( $P=0,431658)$ e a acurácia é significativamente melhor ( $P=0,013004)$ que os valores respectivos do TVS.

A análise dos intervalos de confiança de $95 \%$ da sensibilidade do TVS $(56,41 ; 72,44)$ e TVO $(72,38$; $85,99)$ mostra que eles são levemente superpostos, sinalizando que o novo teste é diferente do antigo, e melhor. Essa mesma análise permite-nos concluir que a especificidade não é significativamente dife- rente, pois os intervalos de confiança de $95 \%$ são altamente superpostos $(35,87 ; 83,72$ para o TVO e 53,69; 95,03 para o TVS).

D os 161 pacientes estudados, 145 (90\%) apresentaram refluxo em, pelo menos, um nível e um sistema na flebografia descendente (refluxo, no mínimo, grau 1, segundo Kistner, ou um nível e um sistema, segundo Raju) e 16 pacientes não apresentaram refluxo. Dos pacientes com refluxo, 112 apresentavam refluxo na veia safena (77\%), com 48 pacientes tendo somente refluxo superficial (33\%). Entre os 56 pacientes com apenas um único nível e sistema envolvido, o TV 0 foi positivo em $79 \%$ e 0 TVS em 55\% ( $P=0,009010)$. Entre os 30 pacientes com refluxo em múltiplos níveis e múltiplos siste- 
mas, o TVO foi positivo em $83 \%$ e o TVS em $77 \%$ $(0,518605)$. $N$ ão houve diferença significativa entre a incidência de refluxo diagnosticada pelo TVO entre pacientes com refluxo em um único nível e único sistema e pacientes com refluxo em múltiplos níveis e múltiplos sistemas ( $P=0,597160)$. H ouve uma ten dência do TVS a não diagnosticar eficientemente as formas de refluxo em um único nível e sistema ( $P=0,051352)$.

\section{D iscussão}

0 primeiro aspecto a ser discutido, quando são analisados novos testes diagnósticos, é o parâmetro usado como padrão-ouro. A flebografiaaindaéusada como método padrão-ouro anatômica e fisiologicamente. $\mathrm{N}$ os 16 pacientes com flebografia descendente negativa para refluxo, o TV 0 foi negativo em 10 epositivo em 6 . Entreesses mesmos pacientes, oTVS foi negativo em 13 e positivo em 3. Em dois pacientes, ambos os testes foram positivos; em quatro pacientes, apenas o TVO foi positivo; e em um paciente, somente o TVS foi positivo. Essa deficiência na especificidade do TVO (mais pacientes com resultados falso-positivos) em relação ao TVS pode ser falsa, e provavelmente o TVO está detectando algum tipo de refluxo que não foi diagnosticado nem pela flebografia (todos os pacientes apresentavam, pelo menos, varicosidades e perfurantes incompetentes diagnosticadas pela flebografia ascendente) nem pelo TVS. A té mesmo os resultados falso-positivos do TVS podem não ser falsos. Então, se um método padrão-ouro impreciso éutilizado, um novo testenão será mel hor que o padrão-ouro, mesmo que esteja mais próximo da verdade $\mathrm{e}^{15}$. Possivel mente, os testes hemodinâmicos têm maior sensibilidade do que a flebografia no diagnóstico de refluxo. 0 bviamente, alta sensibilidade e especificidade são desejadas, mas, na prática, isso é raramente encontrado, indicando que os múltiplos testes paralelos, usados hoje em dia no diagnóstico de doença venosa, ainda sejam necessários.

O utro ponto de discussão éo padrão flebográfico de refluxo. Se apenas a flebografia descendente é considerada no diagnóstico de refluxo, todas as formas de refluxo distal são perdidas. Isso inclui veias perfurantes incompetentes e veias varicosas superficiais, que podem causar refluxo considerável e, conseqüentemente, modificar o padrão pressórico, le- vand o o TVS eo TVO a valores positivos. R esultados semelhantes podem ser obtidos pelo dúplex, que pode ser positivo para refluxo, atémesmo com val vas proximaiscompetentes. M asuda \& Kistner descreveram a classificação de refluxo baseada no padrão da flebografia descendente, que não considera o envolvimento concomitantesuperficial eprofundo e parece ter importância ${ }^{17}$. Com respeito ao refluxo de múltiplos sistemas e múltiplos níveis, el e foi eficientemente diagnosticado pel o TV O e TVS. Entretanto, de forma interessante, o refluxo em um único nível e único sistema é melhor diagnosticado pelo novo TV 0 , o que pode ser considerado outro sinal de melhor sensibilidade.

Em conclusão, a modificação do TVS parece determinar melhor sensibilidade e acurácia sem diminuição significativa na especificidade, provando ser útil no diagnóstico de refluxo.

\section{R eferências}

1. W arren $R$, W hite $E A$, Belcher $C D$. Venous pressures in the saphenous system in normal, varicose and postphlebitic extremities. Surgery. 1949;26:435-45.

2. Pollack AA, W ood EH. V enouspressurein thesaphenousvein in anklein man during exercise and changes in posture. J Appl Physiol. 1949;1:649-53.

3. Arnoldi CC. Venous pressure in patients with valvular incompetence of the veins of the lower limb. Acta C hir Scand. 1966;132:628-45.

4. Raju S, Fredericks R. Evaluation of methods for detecting venousreflux: perspectivesin venousinsufficiency. Arch Surg. 1990;125:1463-7.

5. Kistner RL, FerrisEB, Randhawa G, Kamida C. A method of performing descending venography. J Vasc Surg. 1986;4: 464-8.

6. H erman RJ, N eiman H L, Yao JS, Egan TJ, Bergan JJ, M alave SR. D escending venography: a method of evaluating lower extremity venousvalvular function. R adiology. 1980;137:63-9.

7. M orano JU, Raju S. Chronic venous insufficiency: assessment with descending venography. Radiology. 1990;174:441-4.

8. van Bemmelen PS, Bedford G, Beach K, Strandness DE. $Q$ uantitative segmental evaluation of venous valvular reflux with duplexultrasound scaning. J V asc Surg. 1989;10:425-31.

9. van Bemmelen PS, Beach K, Bedford G, Strandness DE. The mechanisms of venous valve closure. Arch Surg. 1990;125: 617-9.

10. M asuda E, Kistner RL, Eklof B. Prospective study of duplex scanning for venous reflux: comparison of $V$ alsalva and pneumatic cuff techniques in the reverse T rendelenburg and standing positions. J V asc Surg. 1994;20:711-20. 
11. Porter JM , M oneta GL, International C onsensus C ommittee on chronic venous diseases: Reporting standards in venous disease: an update. J V asc Surg. 1995;21:635-45.

12. Raju S, Fredericks R. H emodynamic basis of stasis ulceration a hypothesis. J V asc Surg. 1991;13:491-5.

13. Thomas $L M, M C D$ onald $L M$. Complications of phlebography of the leg. BM J. 1978;2:307-15.

14. Raju S. Valve reconstruction procedures for chronic venous insufficiency. Semin Vasc Surg. 1988;1:101-6.

15. Raju S, Fredericks R. Valve reconstruction procedures for nonobstructive venous insufficiency: rationale, techniques and resultsin 107 procedureswith two to eight year follow-up. J V asc Surg. 1988;7:301-10.

16. Fletcher RH, Fletcher SW, W agner EH. Diagnóstico. In: Fletcher RH , Fletcher SW, W agnerEH . Epidemiologia clínica. Porto Alegre, RS: Artes M édicas; 1991. p. 68-107.
17. M asuda EM, Kistner RL. Prospective comparison of duplex scanning and descending venography in the assessment of venous insufficiency. Am J Surg. 1992;164:254-9.

\section{Correspondência:}

Gilberto G onçalves de Souza

Rua Carlos úlio Becker, 275, Tristeza

CEP 91920-020 - Porto Alegre, RS

Tel.: (51) 3268.9224

E-mail: gsouza@pro.via-rs.com.br 